\title{
Ultrasonic Texture Analysis in Preterm and Term Fetal Lungs
}

\author{
Sleiman R. Ghorayeb ${ }^{1,2,3}$, Zara Rahman', Luis Bracero ${ }^{4,5}$ \\ ${ }^{1}$ School of Engineering and Applied Sciences, Ultrasound Research Laboratory, Hofstra University, Hempstead, NY \\ 11549, USA; \\ ${ }^{2}$ Departments of Radiology and Molecular Medicine, Hofstra Northwell School of Medicine, Hempstead, NY 11549, USA; \\ ${ }^{3}$ Center for Immunology \& Inflammation, The Feinstein Institute for Medical Research, Northwell Health, Manhasset, NY \\ 11030, USA; \\ SGhorayeb@nshs.edu; zrahma4@pride.hofstra.edu \\ ${ }^{4}$ Division of Maternal-Fetal Medicine, Department of Obstetrics and Gynecology, Hofstra Northwell School of Medicine, \\ Southside Hospital, Bay Shore, NY, USA; \\ ${ }^{5}$ Division of Maternal-Fetal Medicine, Department of Obstetrics and Gynecology, Hofstra Northwell School of Medicine, \\ North Shore University Hospital, Manhasset, NY 11549, USA \\ LBracero@Northwell.edu
}

\section{Extended Abstract}

Lung immaturity remains the most common cause of morbidity and mortality in preterm and early term neonates [1]. Rapid, precise and diagnostically sensitive tests of lung maturity are now available but unfortunately, they remain invasive, have low specificity, and remain poor predictors of fetal lung immaturity [2,3]. Heterogeneity, which has been associated with fetal lung maturity, is only grossly detected on grayscale ultrasound. To quantify the extent of heterogeneity associated with fetal lung maturity, we employed a unique, noninvasive technique to determine Dynamic Range (DR) in ultrasound images, and then compared DR in ultrasound of preterm ( $<37$ weeks of gestation) from term ( $\geq 37$ weeks of gestation) fetal lungs by using quantitative texture analysis.

We performed a retrospective evaluation of singleton gestations with reliable dating at $\geq 20$ weeks gestational age between January 2015 and December 2015. A total of 425 fetal lung ultrasound images (313 preterm, 112 term) were analyzed. A region of interest (ROI) was selected in each fetal lung image at the level of the four heart chambers, from an area which appeared most representative of the overall lung tissue and had the least shadow. Ultrasonic tissue heterogeneity (heterogeneity index, HI) was determined for all lung images. This quantification was performed with a custom-made software program [4-6] that uses a dithering technique based on the Floyd-Steinberg algorithm in which the pixels are transformed into a binary map. Regression analysis was used to determine the correlation and functional association between $\mathrm{HI}$ and gestational age. A receiver operating characteristic (ROC) curve was used to identify the optimal HI cut point to differentiate preterm from term fetal lungs. ROC analysis of the data using 0 for the false data and 1 for the true data. Quantitative texture analysis predicted a preterm lung with a sensitivity of $94.7 \%$, a specificity of $96 \%$, using a threshold HI value of 2.105 between the preterm and term fetuses. The positive predictive value and the negative predictive value were determined to be $98.5 \%$ and $86.7 \%$ respectively, with a positive likelihood ratio of 23.6 and a negative likelihood ratio of 0.055 . Further $t$-test analysis on the individual HIs with their respective gestation periods gave a $t$-value of 26.26 with 848 degrees of freedom, which corresponds to a p-value $<0.00001$.

Quantitative ultrasound texture analysis can detect the histologic changes that occur in fetal lung tissue as gestation advances. Specifically, decreased tissue heterogeneity on ultrasound is associated with preterm fetal lung tissue.

\section{References}

[1] P. A. Robertson, S. H. Sniderman, R. K. Laros, Jr., et al, "Neonatal morbidity according to gestational age and birth weight from five tertiary care centers in the United States, 1983 through 1986," Am J Obstet Gynecol, vol. 166, no. $6 \mathrm{Pt}$ 1, pp. 1629-41, 1992.

[2] A. E. Besnard, S. A. Wirjosoekarto, K. A. Broeze, et al, "Lecithin/sphingomyelin ratio and lamellar body count for fetal lung maturity: a meta-analysis," Eur J Obstet Gynecol Reprod Biol, vol. 169, no. 2, pp. 177-83, 2013.

[3] D. G. Grenache and A. M. Gronowski, "Fetal lung maturity," Clin Biochem, vol. 39, no. 1, pp. 1-10, 2006. 
[4] S. R. Ghorayeb, A. Levin, M. Ast, et al, "Sonographic evaluation of knee cartilage defects implanted with preconditioned scaffolds," J. Ultrasound Med, vol. 33, no. 7, pp. 1241-53, 2014.

[5] S. R. Ghorayeb and D. M. Rooney, "Ultrasonic evaluation of bone quality in cadaver ilia," Ann Biomed Eng, vol. 41, no. 5, pp. 939-51, 2013.

[6] S. R. Ghorayeb, N. V. Shah, F. Edobor-Osula, et al, "Ultrasonic assessment of extracellular matrix content in healing Achilles tendon," IEEE Trans Ultrason Ferroelectr Freq Control, vol. 59, no. 4, pp. 694-702, 2012.

[7] S. R. Ghorayeb, C. Hartman, P. Samson, et al, "Ultrasonic evaluation of heterogeneity levels in normal and torsed testes," in Proceedings of the $2^{\text {nd }}$ International Conference on Biomedical Engineering and Systems, Barcelona, Spain, 2015, pp. 297.1-297.4. 